\title{
Influence of Drawing Down on the Properties of Thermotropic Liquid Crystalline Polymer/Polyether Sulfone Composites
}

\author{
Huimin $\mathrm{WANG}_{\mathrm{AN}}{ }^{\dagger}$ Xiaosu $\mathrm{YI}_{\mathrm{I}}{ }^{*}$ and Georg HINRICHSEN ${ }^{* *}$ \\ Department of Chemistry, Shandong University, Jinan 250100, People's Republic of China \\ * Department of Polymer Science and Engineering, Zhejiang University, Hangzhou 310027, \\ People's Republic of China \\ ** Institute of Nonmetallic Materials, Technical University Berlin, \\ Englische Str. 20, D-10587 Berlin, Germany
}

(Received December 2, 1996)

\begin{abstract}
The mechanical, morphological, and thermal properties of composites consisting of a thermotropic liquid crystalline polymer (TLCP) as reinforcing phase in a polyether sulfone (PES) as matrix were investigated. The elastic modulus and tensile strength along the drawing down direction rise with draw down ratio, keeping all other parameters constant. This is attributed to the fibrillation of the TLCP phase in the blend and enhanced orientation of TLCP chains along the drawing down direction during drawing. The fusion heat of the TLCP phase increases continuously with draw down ratio, while the crystal-nematic transition temperature, $T_{\mathrm{m}}$, and glass transition temperature, $T_{\mathrm{g}}$, of the PES matrix are unaffected. KEY WORDS Thermotropic Liquid Crystalline Polymer / Mechanical / Morphological and Thermal Properties / Drawing Down / Orientation /
\end{abstract}

Thermotropic liquid crystalline polymers (TLCPs) offer high levels of thermal and mechanical performance. In the past years, they have been used for preparing in situ composites, ${ }^{1-4}$ by means of blending of the TLCP with a thermoplastic matrix mostly on conventional compounding equipment such as twin-screw or singlescrew extruder with a static mixer. A molten blend of TLCP with thermoplastics improves the processability and mechanical properties because TLCP as a minor component is oriented in the flow direction and can form in situ microfibrils that reinforce the matrix. An additional advantage of such materials is the $100 \%$ recyclability. The materials may be chopped up, melted and reprocessed. ${ }^{2,5}$

In our previous papers, ${ }^{6-8}$ the relationship between the microstructure developed during injection moulding of two blends of TLCP/polycarbonate (PC) and TLCP/ polyether sulfone (PES), respectively, and their mechanical properties were investigated. The major purpose of this paper is to study the effects of the drawing down on the mechanical, morphological and thermal properties of the in situ composites of TLCP/PES.

\section{EXPERIMENTAL}

\section{Materials}

TLCP used was manufactured by Bayer Co. under the trade name KU9231. The detailed composition was not given by the manufacturer. The microstructural studies showed that it behaves as wholly aromatic and nematic polymer. A possible structure is:

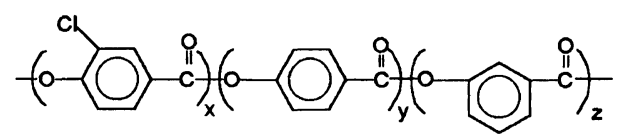

The thermoplastic material utilized was PES with density of $1.37 \mathrm{~g} \mathrm{~cm}^{-3}$ and Vicat softening temperature point above $220^{\circ} \mathrm{C}$, purchased from Jilin University, China. Pellets of TLCP and PES were dried in an air-circulated oven at $120^{\circ} \mathrm{C}$ for at least $24 \mathrm{~h}$ prior to processing. TLCP content in the TLCP/PES blends is 10 and $30 \mathrm{wt} \%$, respectively.

\section{Blending and Processing}

Composite strands were produced by mixing pellets of TLCP and PES in dry state, feeding the mixture through an extruder, and drawing the resulting strands. The extruder used was a $30 \mathrm{~mm}$ co-rotating twin-screw type with a $3 \mathrm{~mm}$ conical die. The downstream barrel temperature was set at $310^{\circ} \mathrm{C}$. Typical drawn strand diameters ranged from 0.8 to $3 \mathrm{~mm}$. Draw down ratio is defined as the ratio of cross-section area of the die and extruded strand.

\section{Mechanical, Morphological, and Thermal Tests}

Mechanical properties of the extruded strands were measured at room temperature at a drawing speed of $10 \mathrm{~mm} \mathrm{~min}^{-1}$ using a Shimadzu DCS (model 200) tensile tester with a gauge length of $30 \mathrm{~mm}$. All reported data points represent the average values of at least three tests. Standard deviation was less than $10 \%$.

The morphology of the extruded strands were obtained by examining the fracture surfaces with a Stereoscan 600 scanning electron microscope.

Differential scanning calorimetry measurements were carried out on a Perkin-Elmer (DSC-7) under nitrogen atmosphere. Sample weight was $\sim 10 \mathrm{mg}$ and heating rate was $10^{\circ} \mathrm{Cmin}^{-1}$. Peak temperature was taken as crystal-nematic transition temperature or melting temperature. In the case of blend samples, the fusion heat of TLCP was obtained by normalizing the measured value by the weight fraction of TLCP in the blend.

\footnotetext{
$\dagger$ To whom correspondence should be addressed.
} 


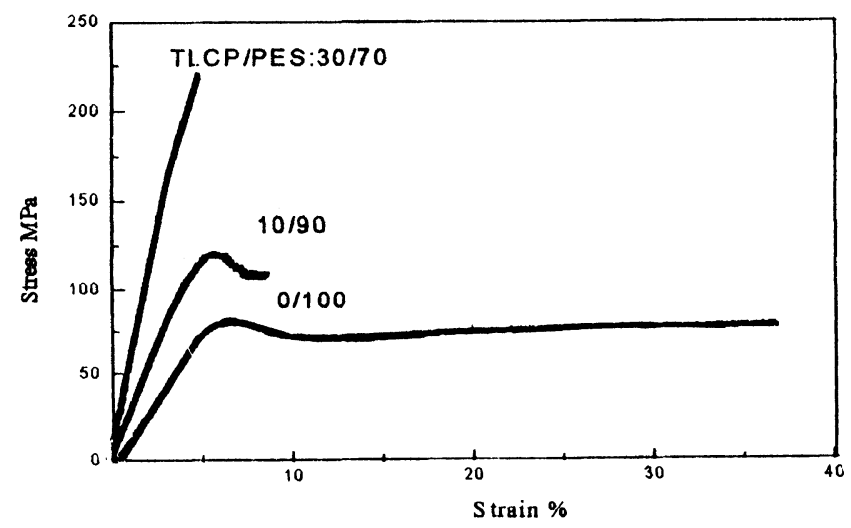

Figure 1. Stress-strain curves of the composite strands with a draw down ratio of 9 .

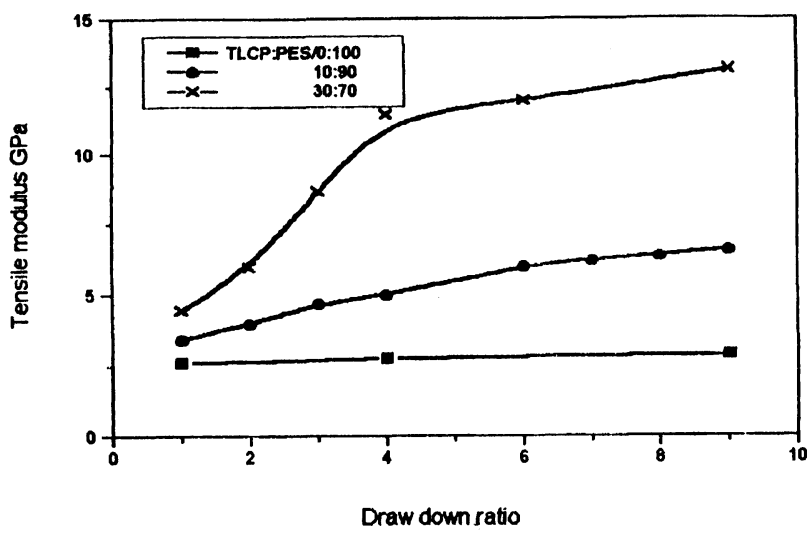

Figure 2. Elastic modulus in dependence on draw down ratio for the TLCP/PES composite strands.

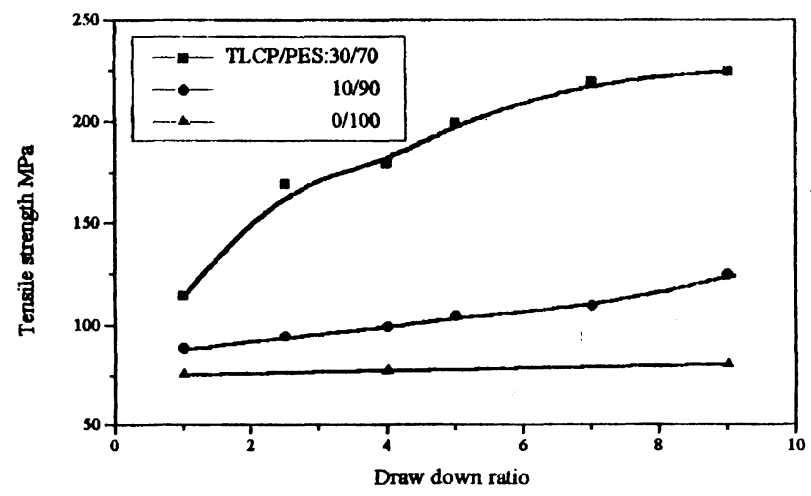

Figure 3. Tensile strength in dependence on draw down ratio for the TLCP/PES composite strands.

\section{RESULTS AND DISCUSSION}

The stress-strain curves for the composite strands with a drawn down ratio of 9 are shown in Figure 1. Strands with $30 \mathrm{wt} \%$ TLCP exhibit no yield point and result in improved tensile strength and modulus. The variation of the elastic modulus of the strands with the draw down ratio is shown in Figure 2. The elastic modulus of pure PES is less affected, while that of the composite strands increases with draw down ratio, but the pattern of increase is different. The strands with $10 \mathrm{wt} \%$ TLCP show slight increase of the modulus with drawn down ratio, while strands with $30 \mathrm{wt} \%$ TLCP indicate a sharp rise.

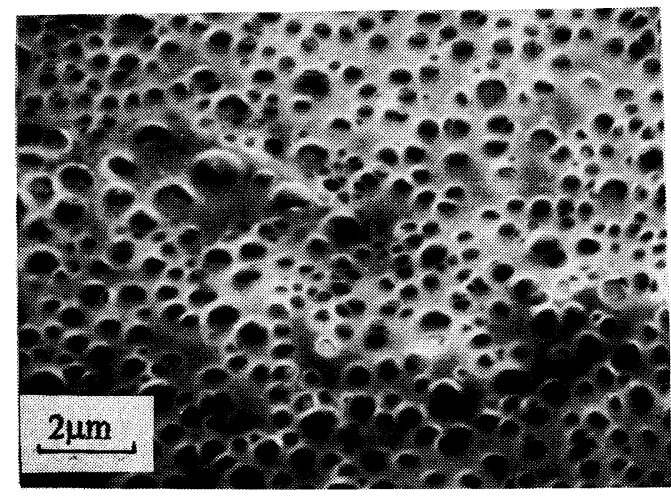

(a) Undrawn

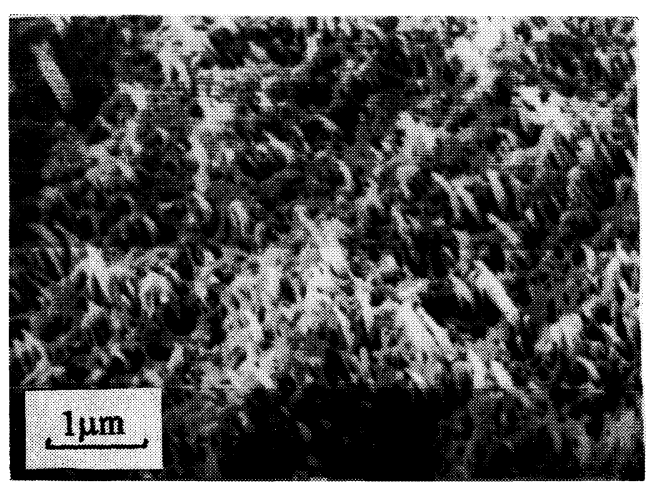

(b) Drawn down

Figure 4. SEM micrographs of the fractures of an undrawn and a drawn down strands of $30 \mathrm{wt} \%$ TLCP blends with a draw down ratio of 9 .

This improvement is caused by the formation of a fibrillar structure of TLCP in the matrix. ${ }^{1,9,10}$ According to the theory of composites, the key to improve stiffness of in situ composites is to promote the TLCP molecular orientation and to develop TLCP microfibrils, although there are other factors affecting the modulus.

The dependence of tensile strength of the drawn down ratio for TLCP/PES composite strands is shown in Figure 3. The tensile strength of the strands with $30 \mathrm{wt} \%$ TLCP increases strongly with draw down ratio up to $\sim 4$ and shows reduced increase above a drawn down ratio of 4 . The tensile strength of the composite strands with $10 \mathrm{wt} \%$ TLCP increases slightly with the draw down ratio.

The development of a fibrillar structure is the key step to achieve TLCP in situ reinforced thermoplastics. However, the generation of the TLCP fibrillar structure is a complex rheological as well as thermal process. ${ }^{11}$ Figure 4 presents SEM micrographs of the fracture surfaces of an undrawn and a drawn down strand with a draw down ratio of 9 for $30 \mathrm{wt} \%$ TLCP. It can clearly be seen that the dispersed TLCP phase appears in a spherical form on the fracture surface of the undrawn sample, while the sample with a draw down ratio of 9 exhibits uniform and well-defined TLCP microfibrils. This shows that the drawing down is crucial to achieve a well-defined TLCP microfibrillar morphology. ${ }^{6}$

The effect of drawing down on the crystallization of the TLCP phase in the blends was revealed by DSC measurements. Changes of the thermal properties with the draw down ratio are depicted in Figures 5-7. The 


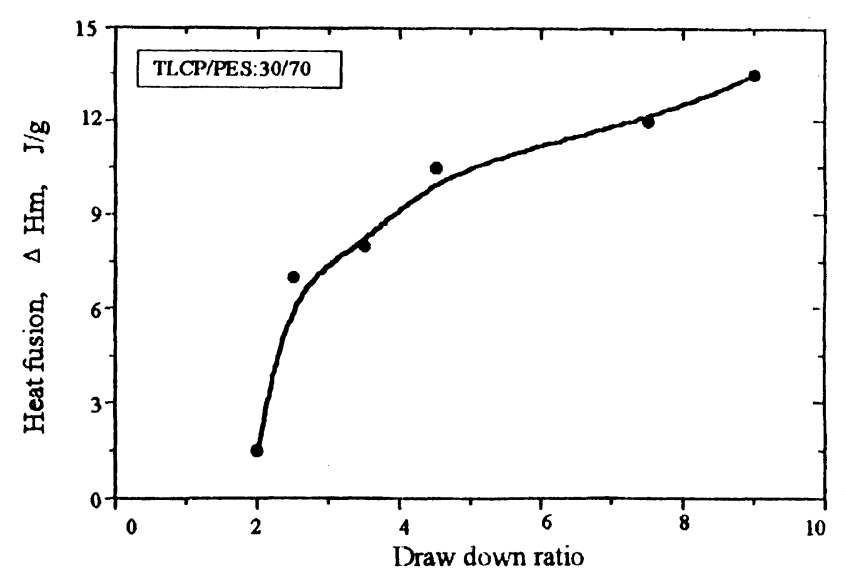

Figure 5. Fusion heat of TLCP in dependence on draw down ratio for $30 \mathrm{wt} \%$ TLCP/PES composite strands.

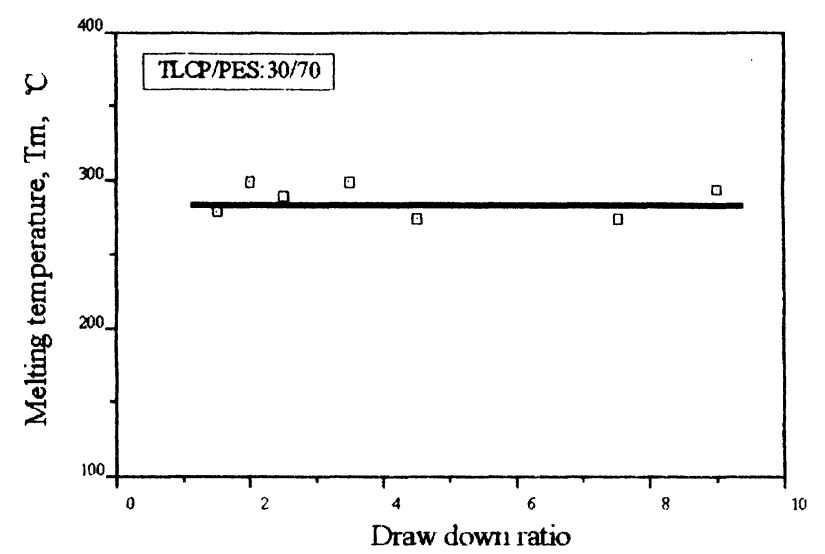

Figure 6. Melting temperature of TLCP in dependence on draw down ratio for $30 \mathrm{wt} \%$ TLCP/PES composite strands.

fusion heat of the TLCP phase increases continuously with draw down ratio. However, the crystal-nematic transition temperature, $T_{\mathrm{m}}$, of TLCP and the glass transition, $T_{\mathrm{g}}$, of the PES matrix are almost unaffected by drawing down. The constant $T_{\mathrm{g}}$ of the PES matrix independent of drawing down ratio indicates that the drawing down used in the present study does not give rise to change of the structure in the glassy PES matrix.

Our experimental results show that, within the limits of experimental error, the "melting" temperature of TLCP remains basically constant and independent of the draw down ratio. Due to the wholly aromatic nature of the KU9231, the change of extension of this (semi)rigid chain during drawing down is much smaller than with flexible chains. For liquid crystalline melts like KU9231, local order still exists even in the unstrained state. Consequently, entropy decrease, $\delta S$, due to drawing down is not as significant for flexible polymers, thus keeping the crystal-nematic transition temperature independent of the draw down ratio. The increase of fusion heat can be explained as $\Delta H_{\mathrm{m}}=T_{\mathrm{m}} \Delta S_{\mathrm{m}}$. The solid state of the TLCP phase becomes more ordered as the drawn down

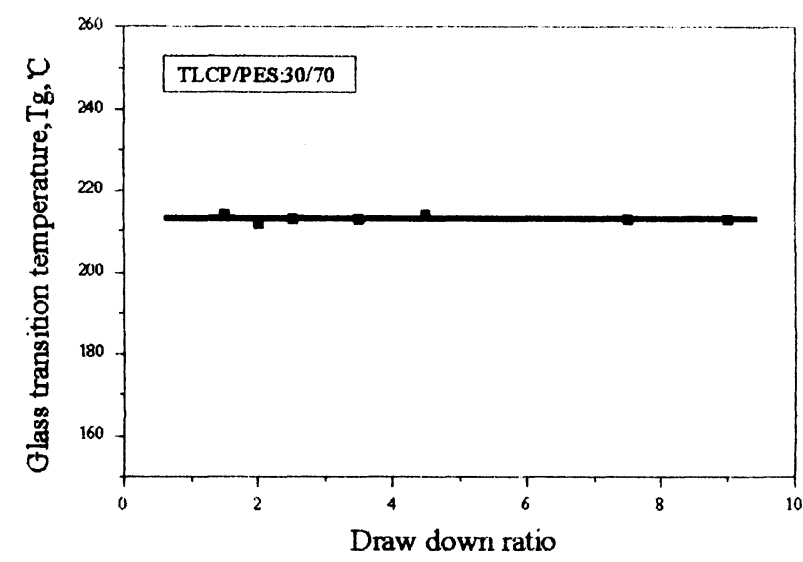

Figure 7. Glass transition temperature of PES in dependence on draw down ratio for $30 \mathrm{wt} \%$ TLCP/PES composite strands.

ratio increases. The entropy difference on the melting increases with the draw down ratio, thus leading to increase of the fusion heat.

\section{CONCLUSIONS}

Drawing down has major effect on structure and hence the properties of the investigated composite strands. It results in fibrillation of the TLCP phase in the blend and increased orientation of the TLCP chains along the drawing down direction. The composite strands with well-defined microfibrils consisting of the $30 \mathrm{wt} \%$ TLCP in the PES matrix, and fabricated by an extrusion and subsequent drawing down, have a high tensile modulus and high tensile strength, compared with the thermoplastic matrix. The fusion heat of the TLCP increases with the draw down ratio, while the crystal-nematic transition temperature, $T_{\mathrm{m}}$, and PES matrix, $T_{\mathrm{g}}$, are essentially unaffected by draw down.

Acknowledgments. The authors would like to thank the National Advanced Materials Committee and the State Education Commission of China for financial support.

\section{REFERENCES}

1. G. Kiss, Polym. Eng. Sci., 27, 410 (1987).

2. B. R. Bassett and A. F. Yee, Polym. Compos., 11, 10 (1990).

3. D. Dutta, F. Fruitwala, A. Kohli, and R. A. Weiss, Polym. Eng. Sci., 30, 1005 (1990).

4. W. Brostow, Polymer, 31, 979 (1990).

5. H. M. Wang and X. S. Yi, J. Zhejiang University, 6, 96 (1994).

6. X. S. Yi, L. S. Wei, and H. M. Wang, Acta Materiae Compositae Sinica, 2 (1994)

7. H. M. Wang and X. S. Yi, Acta Polymerica Sinica, 5, 629 (1993).

8. H. M. Wang and X. S. Yi, Acta Polymerica Sinica, 6, 749 (1993).

9. S. Siegman, A. Dagan, and S. Kenig, Polymer, 26, 1325 (1985).

10. A. Mithal, A. Tayebi, and C. H. Lin, Polym. Eng. Sci., 21, 1533 (1991).

11. K. G. Blizard, C. Federici, O. Federici, and L. L. Chappy, Polym. Eng. Sci., 30, 1442 (1990). 